\title{
O que mudou na incidência da sífilis no estado do Rio de Janeiro de 2009 a 2019
}

\author{
What has changed in the incidence of syphilis in the state of Rio de Janeiro from 2009 to 2019
}

Iara Almeida Adorno ${ }^{\dagger *}$; Bianca Gomes Queiroz ${ }^{\S}$; Thaís Moreira Larall; Renata Duarte Ferreira ${ }^{\square}$; Anne Caroline Barbosa Pires Braga*; Thais Rocha Salim

Como citar esse artigo. Adorno, I.A.; Queiroz, B.G.; Lara, T.M.; Ferreira, R.D.; Braga, A.C.B.P.; Salim, T.R. O que mudou na incidência da sífilis no estado do Rio de Janeiro de 2009 a 2019. Revista de Saúde. 2020 Dez./Mar.; 12 (1): 64-72.

\section{Resumo}

A sífilis é uma doença infectocontagiosa crônica e sistêmica que possui prevenção, diagnóstico e tratamento fácil, rápido e eficaz, mas que, atualmente, vem apresentando elevada taxa de detecção, caracterizando um grave problema de Saúde Pública nacional e global. Este estudo objetiva descrever como a sífilis vem sendo notificada no estado do Rio de Janeiro. Trata-se de um estudo ecológico e descritivo sobre a incidência e distribuição regional da sífilis adquirida, gestacional e congênita no estado do Rio de Janeiro nos anos de 2009 a junho de 2019. Utilizou-se bancos de dados oficiais do SINAN, SINASC, IBGE e da Secretaria de Saúde do Estado do Rio de Janeiro, onde os dados coletados foram caracterizados de acordo com variáveis biológicas, sociais e geográficas. Foram notificados, respectivamente, 55.991, 45.783 e 30.232 casos de sífilis adquirida, gestacional e congênita. Houve predomínio de gestantes entre 20 e 29 anos, pardas, com ensino fundamental incompleto e provenientes da região Metropolitana I. A sífilis gestacional foi mais diagnosticada no primeiro trimestre gestacional, predominando a subclasse latente. Já a congênita apresentou maior incidência em menores de 27 dias, subclasse congênita recente. O momento do diagnóstico materno para sífilis congênita ocorreu principalmente durante o pré-natal. Pôde-se identificar números consideravelmente altos e alarmantes, bem como a incompletude de informações nos bancos de dados. Deste modo, observou-se a necessidade do desenvolvimento e aprimoramento de estratégias de saúde a assistência materno-infantil mais resolutivas visando o controle da sua transmissão, melhoria do diagnóstico e tratamento e a minimização de suas repercussões.

Palavras-chave: Sífilis; Sífilis Congênita; Sífilis Latente; Epidemiologia.

\begin{abstract}
Syphilis is a chronic and systemic infectious disease that has easy, fast and effective prevention, diagnosis and treatment, but which currently has high detection rate, characterizing a serious national and global Public Health problem. This study aims describe how syphilis has been reported in State of Rio de Janeiro. This is an ecological and descriptive study on the incidence and regional distribution of acquired, gestational and congenital syphilis in State of Rio de Janeiro from 2009 to june 2019. Official SINAN, SINASC, IBGE and the Health Department of the State of Rio de Janeiro databases were used, where the data collected were characterized according to biological, social and geographical variables. 55,991, 45,783 and 20,232 cases of acquired, gestational and congenital syphilis were reported, respectively. There was a predominance of pregnant women between 20 and 29 years old, mixed race, with incomplete elementary education and coming from the Metropolitan I region. Gestational syphilis was more diagnosed in the first gestational trimester, with a predominance of the latent subclass. Congenital, on the other hand, had a higher incidence in children younger than 27 days, a recent congenital subclass. The moment of maternal diagnosis for congenital syphilis occurred mainly during prenatal care. One can identify considerably high and alarming numbers, as well as the incompleteness of information in the databases. Thus, there was a need for the development and improvement of health strategies for more effective maternal and child care aimed at controlling their transmission, improving diagnosis and treatment and minimizing their repercussions.
\end{abstract}

Keywords: Syphilis; Congenital Syphilis; Latent Syphilis; Epidemiology.

\section{Introdução}

Dentre as principais moléstias relacionadas às doenças infectocontagiosas, a sífilis (SF) tem se destacado como importante fator agravante da saúde pública nacional e mundial ${ }^{1}$. Ainda que se trate de uma doença prevenível e curável, com métodos diagnósticos de baixo custo e tratamento eficaz, dados da Organização Mundialda Saúde(OMS) apontam que aproximadamente 12 milhões de pessoas se infectam por ano no mundo, sendo, destas, 1 milhão de gestantes ${ }^{1,2,3,4}$.

Trata-se de uma doença crônica, sistêmica, de evolução lenta, exclusiva do ser humano e causada pelo Treponema pallidum, uma bactéria da classe das espiroquetas que possui alta patogenicidade e algumas particularidades, como sensibilidade ao calor e a ambientes secos, sendo também de difícil cultivo em meios artificiais. Seu tratamento é considerado de baixo custo e eficaz se realizado corretamente, portanto o diagnóstico precoce e o acompanhamento da resposta

$\dagger$ Discente do Curso de Medicina da Universidade de Vassouras, Vassouras, RJ, Brasil. ORCID ID: https://orcid.org/0000-0002-8944-0576;

§ Discente do Curso de Medicina da Universidade de Vassouras, Vassouras, RJ, Brasil. ORCID ID: https://orcid.org/0000-0002-6986-5534

|| Discente do Curso de Medicina da Universidade de Vassouras, Vassouras, RJ, Brasil. ORCID ID: https://orcid.org/0000-0003-4709-1045

$\square$ Discente do Curso de Medicina da Universidade de Vassouras, Vassouras, RJ, Brasil. ORCID ID: https://orcid.org/0000-0002-3500-2722

- Discente do Curso de Medicina da Universidade de Vassouras, Vassouras, RJ, Brasil. ORCID ID: https://orcid.org/0000-0001-8012-1336.

\$ Docente do Curso de Medicina da Universidade de Vassouras, Vassouras, RJ, Brasil. ORCID ID: https://orcid.org/0000-0002-2606-7460

* Email de correspondência: iara.enf@hotmail.com

Recebido em: 28/09/20. Aceito em: 08/02/21. 
ao tratamento são de suma importância para o seu controle $e^{5,6,7,8,9}$.

Apesar de possuir vários estágios e oscilar entre períodos sintomáticos e de latência, a SF é classificada em adquirida e congênita, possuindo, respectivamente, transmissão por via sexual e vertical. Esta última decorre da sífilis gestacional (SG), classificação que se refere à gestante infectada, não tratada ou tratada inadequadamente para sífilis adquirida (SA) e que transmite a doença ao feto por via transplacentária ${ }^{1,5,6}$. Destaca-se ainda, mesmo pouco frequente, a transmissão por material perfurocortante, transfusão sanguínea e intraparto, momento no qual a criança entra em contato com secreções e lesões da mãe infectada ${ }^{1,5,6,7,8,9}$.

Com repercussões em diversas especialidades e apresentações clínicas variáveis, o diagnóstico tardio e/ou o tratamento inadequado contribuem para a cronificação e severidade do caso, bem como no acometimento de praticamente todos os órgãos do corpo, principalmente os dos sistemas neurológico, cardiovascular, respiratório e gastrointestinal ${ }^{7,10}$. Segundo a OMS, estima-se que $35 \%$ dos casos não tratados progridem para cura espontânea, 35\% permanecem em latência por toda a vida e, anualmente, ocorrem 2 milhões de casos de $\mathrm{SG}$, dos quais 25\% resultam em aborto espontâneo ou natimorto $2,6,8$. Ademais, quando não tratada, aumenta significativamente o risco de infecção por outras infecções sexualmente transmissíveis (IST) e, especialmente, pelo vírus da imunodeficiência humana $(\mathrm{HIV})^{6,7}$.

Segundo Tavares (2015), cerca de $70 \%$ a $100 \%$ das transmissões materno-infantis ocorre nos primeiros quatro anos de $\mathrm{SA}$ e, destas, 30\% resultam em sífilis congênita (SC). A OMS afirma que a $\mathrm{SC}$, que deveria ser diagnosticada e adequadamente tratada no pré-natal $(\mathrm{PN})$, tem mortalidade superior a $50 \%$, contribui com até um quarto de todos os natimortos e cerca de $11 \%$ das mortes neonatais. Em 2009 foram contabilizados aproximadamente 2,6 milhões de natimortos e 3,1 milhões de óbitos em menores de 1 ano em decorrência da $\mathrm{SC}^{3,5,6,8}$.

Para fins de vigilância epidemiológica, a SC tornou-se doença de notificação compulsória no Brasil a partir de 1986, seguida da SG em 2005 e, recentemente, em 2010, da SA ${ }^{5,11,12}$. Segundo o Boletim Epidemiológico de Sífilis 2019, no ano de 2018 foram notificados 15.537 casos de SA, 9.234 de SG, 4.171 de SC e 52 óbitos por SC apenas no estado do Rio de Janeiro ${ }^{9,4}$. Entretanto, a SF vem sendo subnotificada em grande escala no Brasil e em outros países e, em consonância, seu número também tem sofrido um aumento significativo, o que requer atenção da população e dos serviços de saúde como um todo ${ }^{7,11,12,13}$.

Em decorrência da carência de estudos epidemiológicos abordando esta temática justifica-se o incentivo à realização de pesquisas que contribuam para a identificação da epidemiologia local de uma dada população, possibilitando, desta forma, o aprimoramento do planejamento, das ações e intervenções em saúde visando o controle efetivo deste agravo ${ }^{14,15}$. Trilhando este caminho, o presente estudo objetiva descrever como a sífilis vem sendo notificada no estado do Rio de Janeiro entre o período de 2009 e 2019.

\section{Material e Método}

Trata-se de um estudo ecológico e descritivo sobre a incidência da sífilis congênita, gestacional e adquirida no estado do Rio de Janeiro nos anos de 2009 a 2019. Foram utilizados bancos de dados oficiais como o Sistema de Informação de Agravos de Notificação (SINAN) e o Sistema de Informações sobre Nascidos Vivos (SINASC) dos municípios do estado do Rio de Janeiro de janeiro de 2009 a 30 de junho de 2019. O primeiro, decorrente dos registros de notificação e investigação de agravos da lista de doenças de notificação compulsória, varia de acordo com os problemas mais significativos da epidemiologia de determinado município ou estado e possibilita o diagnóstico da incidência e prevalência das doenças que acometem a população. Já o segundo compreende dados referentes aos nascimentos declarados pelo país, disponibilizando ainda informações importantes quanto à saúde da mulher gestante e do recém-nascido ${ }^{16}$.

Ambos os sistemas convergem como ferramenta de planejamento de ação, gestão e intervenção na saúde, de modo a permitir além do acesso à informação, que estratégias sejam traçadas para o melhor enfrentamento aos agravos que predominam de um modo geral e específico em determinada área geográfica, o que, por sua vez, possibilita que haja melhoria da qualidade do serviço prestado e do resultado esperado, independente da esfera e da complexidade do mesmo. Trata-se, portanto, de sistemas capazes de subsidiar a avaliação do impacto do planejamento e suas respectivas intervenções.

O SINAN, por ser composto pelas fichas de notificação compulsória, contém informações clínicas e sociodemográficas fornecidas pelos serviços de saúde. Desta forma, além dos casos de residentes locais, também possui registros de casos cujo atendimento/ diagnóstico foi realizado no estado independente do local de residência. As informações foram subdivididas em macrorregiões de saúde do estado mediante o Plano Diretor de Regionalização de $2013^{17}$.

As variáveis disponíveis quanto a SG foram: (1) número de casos, (2) faixa etária, (3) escolaridade, (4) raça, (5) idade gestacional, (6) classificação clínica e (7) esquema de tratamento. As variáveis relacionadas às crianças com SC foram (1) número de casos, (2) óbitos em menores de um ano, (3) idade ao diagnóstico e (4) diagnóstico final. Já as variáveis de $\mathrm{SC}$ relacionadas à 
mãe foram: (1) faixa etária, (2) escolaridade, (3) raça, (4) realização de pré-natal, (5) momento do diagnóstico da SF materna, (6) esquema de tratamento e (7) tratamento do parceiro. Para a SA utilizou-se o (1) número de casos. Após a obtenção dos dados, realizou-se a análise estatística por meio do programa Microsoft Excel ${ }^{18}$.

Para o cálculo das taxas de incidência de SG e $\mathrm{SC}$ utilizou-se número de casos notificados no ano estudado dividido pelo total de nascidos vivos (NV) do estado no mesmo ano e o valor multiplicado por mil. Para a taxa de incidência de SA, o número de casos foi dividido pelo número de habitantes do respectivo ano e multiplicado por cem mil. O cálculo da redução percentual das taxas de incidência se deu pela subtração do valor inicial e final das taxas de incidência, referente aos anos estudados, cujo resultado foi dividido pelo valor inicial e multiplicado por cem. Foram calculadas a taxa de mortalidade infantil de SC por $100.000 \mathrm{NV}$ com dados da Secretaria Estadual de Saúde do Rio de Janeiro sobre causa básica de óbito (CID10: SC) ${ }^{19}$.

Compondo um dos quatro estados da região Sudeste do Brasil, o Estado do Rio de Janeiro, segundo o último censo de 2019 do Instituto Brasileiro de Geografia e Estatística (IBGE), possui área territorial de $43.750 .427 \mathrm{~km}^{2}$, população estimada de 17.264 .943 habitantes e densidade demográfica de 365,23 hab/ $\mathrm{km}^{220}$.

O estado é dividido em 92 municípios distribuídos entre nove regionais de saúde responsáveis pela gestão dos serviços em saúde, a saber: Baía da Ilha Grande, Baixada Litorânea, Centro-Sul, Médio Paraíba, Metropolitana I, Metropolitana II, Noroeste, Norte e Serrana. A Tabela 1 descreve os municípios pertencentes a cada regional de saúde ${ }^{17,20}$.

Por se tratar de dados secundários e de livre acesso este estudo garantiu a preservação da identidade dos indivíduos respeitando os preceitos éticos da Resolução do Conselho Nacional de Saúde (CNS) nº 466, de 12 de dezembro de 2012, que aborda pesquisas envolvendo seres humanos.

\section{Resultados}

No período de janeiro de 2009 a junho de 2019 foram registrados no estado do Rio de Janeiro 45.783 casos de SG e 30.232 de SC, bem como 55.991 casos de SA de 2010 a 2019. O ano com maior taxa de incidência para SA e SG foi $2018(7,42$ e 41,87) e para SC foi 2017 $(19,30)$. Houve aumento da incidência de SF em todas as variáveis analisadas, onde observou-se um crescimento de 195\% na taxa de incidência de SC entre os anos de

Tabela 1. Regionais de saúde o Estado do Rio de Janeiro.

\begin{tabular}{|c|c|}
\hline \multicolumn{2}{|l|}{ Baía da Ilha } \\
\hline Grande & Angra dos Reis, Mangaratiba, Paraty. \\
\hline Baix & a, Armação dos Búzios, Arraial do Cabo, Cabo Frio, Casimiro de \\
\hline Litorânea & Abreu, Iguaba Grande, Rio das Ostras, São Pedro da Aldeia, Saquarema. \\
\hline Centro-Sul & $\begin{array}{l}\text { Areal, Comendador Levy Gasparian, Engenheiro Paulo de Frontin, } \\
\text { Mendes, Miguel Pereira, Paracambi, Paraíba do Sul, Paty do Alferes, } \\
\text { Sapucaia, Três Rios, Vassouras. }\end{array}$ \\
\hline Médio & Barra do Piraí, Barra Mansa, Itatiaia, Pinheiral, Piraí, Porto Real, Quatis, \\
\hline Paraíba & Resende, Rio Claro, Rio das Flores, Valença, Volta Redonda. \\
\hline $\begin{array}{l}\text { Metropolitana } \\
\text { I }\end{array}$ & $\begin{array}{l}\text { Belford Roxo, Duque de Caxias, Itaguaí, Japeri, Magé, Mesquita, } \\
\text { Nilópolis, Nova Iguaçu, Queimados, Rio de Janeiro, São João de Meriti, } \\
\text { Seropédica. }\end{array}$ \\
\hline \multicolumn{2}{|l|}{ Metropolitana } \\
\hline II & Itaboraí, Maricá, Niterói, Rio Bonito, São Gonçalo, Silva Jardim, Tanguá. \\
\hline Noroe & $\begin{array}{l}\text { Aperibé, Bom Jesus de Itabapoana, Cambuci, Cardoso Moreira, Italva, } \\
\text { Itaocara, Itaperuna, Laje do Muriaé, Miracema, Natividade, Porciúncula, } \\
\text { Santo Antônio de Pádua, São José de Ubá, Varre-Sai. }\end{array}$ \\
\hline Norte & $\begin{array}{l}\text { Campos dos Goytacazes, Carapebus, Conceição de Macabu, Macaé, } \\
\text { Quissamã, São Fidélis, São Francisco de Itabapoana, São João da Barra. }\end{array}$ \\
\hline & $\begin{array}{l}\text { Bom Jardim, Cachoeiras de Macacu, Cantagalo, Carmo, Cordeiro, Duas } \\
\text { Barras, Guapimirim, Macuco, Nova Friburgo, Petrópolis, Santa Maria } \\
\text { Madalena, São José do Vale do Rio Preto, São Sebastião do Alto, } \\
\text { Sumidouro, Teresópolis, Trajano de Moraes. }\end{array}$ \\
\hline
\end{tabular}


2009 e 2018 e de $87 \%$ quando comparado 2009 aos seis meses de registro disponíveis de 2019 . No que tange a SG, o aumento foi equivalente a $880 \%$ entre 2009 e 2018 e $758 \%$ entre 2009 e 2019; já a SA apresentou uma alta doze vezes maior ao número de notificações entre 2010 e 2018 e acréscimo de $394 \%$ entre 2010 e 2019 (Figura 1). $\mathrm{O}$ ano com menor quantitativo de notificações em SG e SC foi 2009 e 2010 para SA.

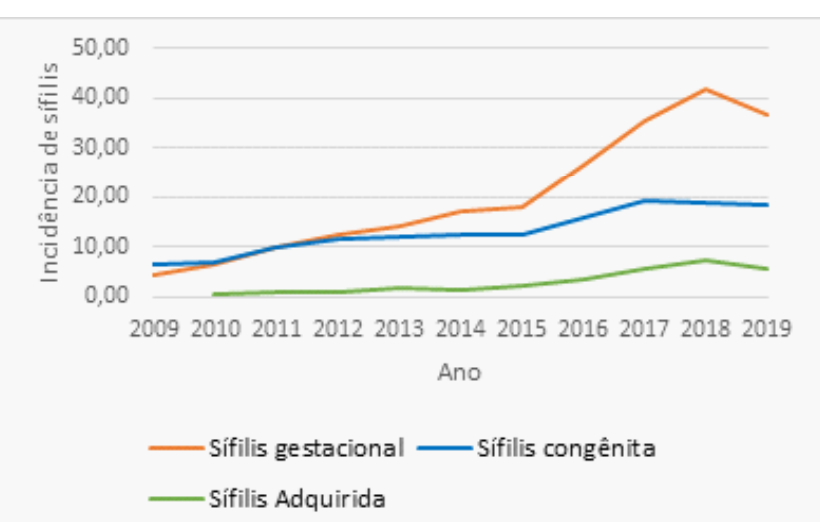

Figura 1. Taxa de incidência da sífilis gestacional e congênita por 1.000 nascidos vivos de 2009 a junho de 2019; e sífilis adquirida por 100.000 habitantes de 2010 a junho de 2019 no Estado do Rio de Janeiro.

Quanto aos casos de SG, no que se refere às características sociodemográficas, as maiores taxas de notificação ocorreram nas regiões Metropolitana I $(78,89 \%)$ e Metropolitana II (7,86\%). A maior incidência foi de gestantes entre 20 e 29 anos (52,97\%), com ensino fundamental incompleto $(26,62 \%)$ e pardas $(43,43 \%)$ (Tabela 2). As regiões Noroeste e Baía da Ilha Grande apresentaram os menores quantitativos, $0,52 \%$ e $0,60 \%$, respectivamente.

O diagnóstico se deu principalmente no primeiro $(34,01 \%)$ (PTG) e terceiro $(30,33 \%)$ (TTG) trimestre gestacional, onde $24,77 \%$ das gestantes apresentaram sífilis latente e $20,81 \%$ sífilis primária. Observou-se que $82,92 \%$ das mulheres apresentavam-se entre $10 \mathrm{e}$ 29 anos. Nota-se, portanto, o perfil de mulheres cada vez mais jovens contraindo a patologia em questão, das quais $40,77 \%$ são analfabetas ou possuem ensino fundamental e médio incompletos, caracterizando uma classe social marginalizada e de provável baixa renda.

É importante frisar o elevado índice de dados ignorados quanto à escolaridade (37,00\%) e classificação clínica (37,52\%), informações fundamentais para melhor identificação e conhecimento do tipo e da qualidade do atendimento prestado, bem como do perfil da população acometida pela doença.

Apesar do SINAN disponibilizar o esquema de tratamento da SG apenas entre 2015 e 2018, correspondendo a 27.286 dos 45.783 dos casos, pôdese observar que $93,61 \%$ das gestantes foram tratadas com penicilina. No entanto, os demais 18.497 casos (40,40\%) referentes ao período de 2009 a 2014 e 2019 não puderam ser analisados.

Tabela 2. Características sociodemográficas e clínicas da sífilis gestacional no Estado do Rio de Janeiro, 2009 a junho de 2019.

\begin{tabular}{|c|c|c|}
\hline Variáveis & $\mathrm{N}=\mathbf{4 5 . 7 8 3}$ & $\%$ \\
\hline \multicolumn{3}{|l|}{ Faixa Etária $^{1}$} \\
\hline 10 a 19 anos & 13.380 & 29,95 \\
\hline 20 a 29 anos & 24.253 & 52,97 \\
\hline 30 a 39 anos & 7.292 & 15,92 \\
\hline 40 anos ou mais & 779 & 1,70 \\
\hline Ignorado & 13 & 0,02 \\
\hline \multicolumn{3}{|l|}{ Escolaridade } \\
\hline Analfabeto & 714 & 1,55 \\
\hline Fundamental incompleto & 12.191 & 26,62 \\
\hline Fundamental completo & 4.231 & 9,24 \\
\hline Médio incompleto & 5.773 & 12,60 \\
\hline Médio completo & 5.366 & 11,72 \\
\hline Superior Incompleto & 376 & 0,82 \\
\hline Superior Completo & 192 & 0,41 \\
\hline Ignorado & 16.940 & 37,00 \\
\hline \multicolumn{3}{|l|}{ Cor da pele } \\
\hline Branca & 9.114 & 19,90 \\
\hline Preta & 9.294 & 20,30 \\
\hline Amarela & 480 & 1,04 \\
\hline Parda & 19.885 & 43,43 \\
\hline Indígena & 61 & 0,13 \\
\hline Ignorada & 6.949 & 15,17 \\
\hline \multicolumn{3}{|l|}{ Idade Gestacional } \\
\hline $1^{\circ}$ Trimestre & 15.572 & 34,01 \\
\hline $2^{\circ}$ Trimestre & 11.213 & 24,49 \\
\hline $3^{\circ}$ Trimestre & 13.886 & 30,33 \\
\hline Ignorada & 5.112 & 11,16 \\
\hline \multicolumn{3}{|l|}{ Classificação Clínica } \\
\hline Sífilis Primária & 9.530 & 20,81 \\
\hline Sífilis Secundária & 1.361 & 2,97 \\
\hline Sífilis Terciária & 6.368 & 13,90 \\
\hline Sífilis Latente & 11.345 & 24,77 \\
\hline Ignorado & 17.179 & 37,52 \\
\hline \multicolumn{3}{|l|}{$\begin{array}{l}\text { Esquema de } \\
\text { Tratamento }^{2}\end{array}$} \\
\hline Penicilina & 25.543 & 93,61 \\
\hline Outro esquema & 179 & 0,65 \\
\hline Não realizado & 728 & 2,66 \\
\hline Ignorado & 836 & 3,06 \\
\hline \multicolumn{3}{|l|}{ Regional do Estado ${ }^{3}$} \\
\hline Baía da Ilha Grande & 275 & 0,60 \\
\hline Baixada Litorânea & 1.331 & 2,90 \\
\hline Médio Paraíba & 1.459 & 0,86 \\
\hline Centro Sul & 397 & 3,18 \\
\hline Metropolitana I & 36.121 & 78,89 \\
\hline Metropolitana II & 3.601 & 7,86 \\
\hline Noroeste & 241 & 0,52 \\
\hline Norte & 1.117 & 2,43 \\
\hline Serrana & 1.240 & 2,70 \\
\hline
\end{tabular}


Com relação às variáveis maternas de $\mathrm{SC}$, também houve prevalência de mulheres entre 20 e 29 anos $(52,62 \%)$, pardas $(50,54 \%)$ e com ensino fundamental incompleto $(30,53 \%)$. A realização de pré-natal ocorreu em $76,47 \%$ dos casos, porém apenas $48,55 \%$ dos diagnósticos de sífilis materna foram realizados durante o $\mathrm{PN}$, conflitando com $39,00 \%$ feitos no momento do parto ou curetagem. O esquema de tratamento das mães de crianças com SC foi considerado inadequado, não realizado ou ignorado em 51,08\%, $30,03 \%$ e $15,43 \%$ dos casos, respectivamente. Desta forma, verificou-se um valor inferior a $4 \%$ das mães recebendo tratamento adequado. Além disso, quanto

Tabela 3. Características sociodemográficas e clínicas de sífilis congênita segundo dados maternos de 2009 a junho de 2019.

\begin{tabular}{|c|c|c|}
\hline Variáveis & $\mathrm{N}=\mathbf{3 0 . 2 3 2}$ & $\%$ \\
\hline \multicolumn{3}{|l|}{ Faixa Etária } \\
\hline 10 a 19 anos & 8.334 & 27,56 \\
\hline 20 a 29 anos & 15.911 & 52,62 \\
\hline 30 a 39 anos & 4.454 & 14,73 \\
\hline 40 anos ou mais & 435 & 1,43 \\
\hline Ignorado & 1.098 & 3,63 \\
\hline \multicolumn{3}{|l|}{ Escolaridade } \\
\hline Analfabeto & 89 & 0,29 \\
\hline Fundamental incompleto & 9.231 & 30,53 \\
\hline Fundamental completo & 2.976 & 9,84 \\
\hline Médio incompleto & 3.164 & 10,46 \\
\hline Médio completo & 3.173 & 10,49 \\
\hline Superior Incompleto & 174 & 0,57 \\
\hline Superior Completo & 116 & 0,38 \\
\hline Ignorado & 11.309 & 37,40 \\
\hline \multicolumn{3}{|l|}{ Cor da pele } \\
\hline Branca & 4.817 & 15,93 \\
\hline Preta & 5.455 & 18,04 \\
\hline Amarela & 102 & 0,33 \\
\hline Parda & 15.280 & 50,54 \\
\hline Indígena & 9 & 0,02 \\
\hline Ignorada & 4.569 & 15,11 \\
\hline \multicolumn{3}{|l|}{ Realização de pré-natal } \\
\hline Sim & 23.121 & 76,47 \\
\hline Não & 4.528 & 14,97 \\
\hline Ignorado & 2.583 & 8,54 \\
\hline \multicolumn{3}{|l|}{$\begin{array}{l}\text { Momento do diagnóstico } \\
\text { da sífilis materna }\end{array}$} \\
\hline Durante o pré-natal & 14.680 & 48,55 \\
\hline $\begin{array}{l}\text { No momento do } \\
\text { parto/curetagem }\end{array}$ & 11.791 & 39,00 \\
\hline Após o parto & 1.810 & 5,98 \\
\hline Não realizado & 131 & 0,43 \\
\hline Ignorado & 1.820 & 6,02 \\
\hline \multicolumn{3}{|l|}{$\begin{array}{l}\text { Esquema de tratamento } \\
\text { materno }\end{array}$} \\
\hline Adequado & 1.042 & 3,44 \\
\hline Inadequado & 15.444 & 51,08 \\
\hline Não Realizado & 9.081 & 30,03 \\
\hline Ignorado & 4.665 & 15,43 \\
\hline \multicolumn{3}{|l|}{ Parceiro tratado } \\
\hline Sim & 3.334 & 11,02 \\
\hline Não & 16.859 & 55,76 \\
\hline Ignorado & 10.039 & 33,20 \\
\hline
\end{tabular}

ao tratamento dos parceiros destas mães, $33,20 \%$ dos dados foram ignorados e apenas $11,02 \%$ tratados para a doença (Tabela 3).

Os abortos por SC computaram apenas 3,26\% dos casos. Novamente, a maior incidência ocorreu nas regiões Metropolitanas I e II e a menor nas Noroeste, Baía da Ilha Grande e Centro Sul (Tabela 4).

Entre os 467 óbitos por SC em menores de um ano, de 2009 a $2018,89,07 \%$ se sucederam até os 27 dias de vida, com o sexo masculino $(57,17 \%)$ e a raça parda $(61,88 \%)$ mais incidentes. Os anos com maior mortalidade foram $2015(13,91 \%)$ e $2017(13,49 \%)$, já 2009 e 2010 obtiveram menor número com 3,64\% e $5,99 \%$ do total. Percebe-se um aumento de $182 \%$ no quantitativo de óbitos quando comparados os anos de 2009 e 2015 (Figura 2). Ainda, apesar da população não possuir dados que totalizem o número de óbitos em 2019, devido à indisponibilidade da informação no sistema, o valor referente a seis meses já supera mais da metade dos casos de 2015.

Tabela 4. Características diagnósticas de sífilis congênita por número de diagnóstico no estado entre 2009 e junho de 2019.

\begin{tabular}{lcc}
\hline Variáveis & $\mathbf{N}=\mathbf{3 0 . 2 3 2}$ & $\mathbf{\%}$ \\
\hline Idade da criança $^{1}$ & & \\
\hline 1 a 27 dias & 29.737 & 98,36 \\
\hline 28 dias a 1 ano & 367 & 1,21 \\
\hline Maiores de 1 ano & 91 & 0,30 \\
\hline Ignorado & 35 & 0,11 \\
\hline Diagnóstico Final & & \\
\hline Sífilis congênita recente & 28.197 & 93,26 \\
\hline Sífilis congênita tardia & 62 & 0,20 \\
\hline Aborto por sífilis & 987 & 3,26 \\
\hline Natimorto por sífilis & 986 & 3,26 \\
\hline Regional do Estado & \\
\hline Baía da Ilha Grande & 147 & 0,48 \\
\hline Baixada Litorânea & 605 & 2,00 \\
\hline Médio Paraíba & 780 & 2,58 \\
\hline Centro Sul & 147 & 0,48 \\
\hline Metropolitana I & 23.314 & 77,11 \\
\hline Metropolitana II & 3.822 & 12,64 \\
\hline Noroeste & 103 & 0,34 \\
\hline Norte & 403 & 1,33 \\
\hline Serrana & & \\
\hline & & \\
\hline
\end{tabular}

${ }^{1}$ Esta informação é compatível com os casos suspeitos, o restante dos dados apresentados é referente aos casos confirmados.

2112 casos não foram relatados por Regional do Estado. 


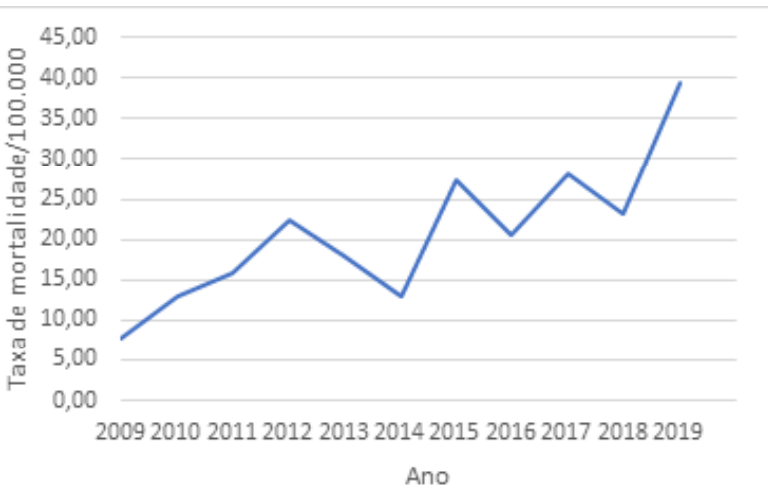

Figura 2. Taxa de mortalidade infantil por sífilis congênita no estado do Rio de Janeiro de 2009 a junho de 2019 por 100.000 nascidos vivos.

\section{Discussão}

Este estudo observou uma ascensão da incidência de SF no estado do Rio de Janeiro e, segundo a OMS, tal realidade não se faz diferente em outras regiões do Brasil e em outros países ${ }^{2,4,21}$. Padrão semelhante foi registrado em estudos realizados no estado do Paraná ${ }^{15}$, Santa Catarina ${ }^{21}$, Espírito Santo ${ }^{22}$, Tocantins $^{23}$, Rio Grande do Norte ${ }^{24}$, em Caxias (MA) ${ }^{14}$, Maringá (PR) ${ }^{25}$, Santa Maria (RS) ${ }^{26}$, no Vale do Paraíba $(\mathrm{SP})^{27}$, bem como em Barcelona (Espanha) ${ }^{28}$ e nos Estados Unidos ${ }^{29}$. Desta forma, a alta incidência de notificações alerta para a magnitude deste problema de saúde pública e reforça a urgência no desenvolvimento de estratégias de saúde mais efetivas para melhoria na prevenção, no atendimento, diagnóstico e manejo dos infectados. Para Padovani (2018), essa estatística além de alarmante pode estar subestimada, visto que o Brasil notifica apenas $32 \%$ e $17,4 \%$ dos casos de SG e SC, respectivamente ${ }^{25}$. Logo, frente ao elevado número de dados ignorados e não informados, torna-se também de grande valia a capacitação contínua e sistemática dos profissionais de saúde quanto ao correto preenchimento das notificações, visando à disponibilidade de informações mais fidedignas para a epidemiologia em questão e a redução das subnotificações.

A taxa de detecção de SA e SG, no ano de 2018, superou os 75,8 (por 100.000 habitantes) e 24,1 casos (por $1.000 \mathrm{NV}$ ) do país no mesmo ano, respectivamente4. Em 2008 , estimativas apontaram que $44,3 \%, 39,3 \%$ e $7,8 \%$ das grávidas apresentavam-se com SG na Ásia, África e nas Américas, respectivamente ${ }^{24}$. Estudos equivalentes levantam questões que podem justificar tal quantitativo, a saber: ações inefetivas de educação em saúde; não realização ou inadequação do PN preconizado para SF; tratamento inadequado ou inexistente para cada fase clínica, tanto na gestante quanto no parceiro; má gestão dos recursos de saúde; melhoria da qualidade dos dados notificados do SINAN e falha na atenção à saúde da mulher ${ }^{14,15,21,22}$.

Acredita-se que o grande contingente populacional e, consequentemente, o maior número de serviços de saúde disponíveis justifiquem a elevada ocorrência dos registros em todos anos nas regiões Metropolitanas I e II. Entretanto, quanto as demais regiões que apresentaram baixas taxas de notificação, é possível supor, pela discrepância dos números, quando comparadas com as metropolitanas, que haja subnotificação e/ou falha no diagnóstico e manejo da doença, o que direciona à necessidade de implementação de políticas públicas mais resolutivas e efetivas pelos gestores de saúde. Estudo realizado em 2014 no Rio Grande do Norte identificou que municípios com menos recursos possuíam maior probabilidade e tendência às subnotificações, o que pode explicar os resultados observados $^{22,30}$.

Segundo o Boletim Epidemiológico de Sífilis de 2019, outra razão para o aumento dos casos pode se dar pela alteração no critério de definição dos casos, que, a partir de 2017 , passou a considerar a notificação de SG durante o pré-natal, parto e puerpério. No entanto, a situação é muito grave, pois, em 2018 , estado e cidade do Rio de Janeiro apresentaram, respectivamente, taxas de detecção para SA $(90,5$ e 105,9), SG $(41,4$ e 51,5) e SC $(18,7$ e 14,0$)$ que superaram a já então elevada média nacional $(75,8 / 100.000$ hab, 21,4/1.000 NV e 9,0/1.000 NV) ${ }^{4}$.

Em 2018, dos casos de SG no Brasil, prevaleceram gestantes com faixa etária entre 20 e 29 anos $(52,5 \%)$, ensino médio incompleto $(51,5 \%)$, pardas $(50,8 \%)$ e brancas $(28,6 \%)$, variáveis que quase se repetem quanto as mães de crianças com $\mathrm{SC}$, que se apresentaram entre 20 e 29 anos $(53,6 \%)$, com ensino fundamental incompleto $(22,2 \%)$, pardas $(58,4 \%)$ e brancas $(23,4 \%)^{4}$. Logo, os resultados deste estudo estão em consonância com os nacionais e estaduais no que tange a idade, escolaridade e raça das mulheres diagnosticadas com $\mathrm{SF}^{4,23,24}$. Quanto à $\mathrm{SG}$, a maior frequência de gestantes jovens entre 20 e 35 anos e com baixa escolaridade corrobora com outros estudos $^{4,14,26,30,31,32,33}$. Dois estudos identificaram maior prevalência de SG em grávidas com idade inferior a 20 anos, o que fortalece os $82,18 \%$ dos casos identificados entre 10 e 29 anos $^{14,25}$. Portanto, a vulnerabilidade imposta pela imaturidade etária somada ao início da vida sexual cada vez mais precoce entre adolescentes e aliada à baixa escolaridade, ao não uso de métodos preventivos de gravidez e IST, bem como à inadequação ou não realização do tratamento das gestantes e seus parceiros são pontuados como fatores agravantes e causais. Ainda que seja autodeclarada, a raça parda também é frequente em outras pesquisas, apesar do predomínio de mulheres pretas nos Estados Unidos e não brancas de um modo geral no Brasil $1^{14,15,27,29,30}$. Apesar de se tratar de uma 
doença sem predileção pela cor da pele, questiona-se se a incidência e probabilidade de se contrair SF continue acometendo mulheres marginalizadas e vulneráveis social e economicamente, uma vez que há relação com o baixo nível socioeconômico, escolar e difícil acesso à saúde de qualidade $27,31,32,33$.

Segundo Brasil (2019), em 2018, também houve predomínio de $\mathrm{SG}$ no $\mathrm{PTG}$ e TTG. A região Sudeste do país registrou $44,7 \%$ do total de SG no PTG4. Em contrapartida, outros estudos apontam maior registro no TTG e segundo trimestre gestacional (STG), o que caracteriza, portanto, um diagnóstico ou início de PN tardio ${ }^{14,15,23}$. Quanto a incidência da classificação clínica de SF primária frente à SF latente, resultados semelhantes são relatados em outras pesquisas e um estudo nacional de 2005 a 2016 corrobora com o achado, mas levanta a possibilidade de erros e divergências na classificação $0^{4,15,25}$.

Sabe-se que a grande maioria das gestantes não apresenta manifestações clínicas da SF e, aliado ao curto período sintomático da fase primária, estes fatores desafiam e dificultam o diagnóstico precoce. Portanto, frente a impossibilidade de se firmar a fase diagnóstica é preconizado que a classificação mais adequada seja de SF latente indeterminada, exceto quando conhecido o tempo de evolução da doença. Ressalta-se que o erro na classificação seja fator limitante para o correto esquema de tratamento ${ }^{6,10,25}$. Ainda que o tratamento preconizado esteja sendo realizado majoritariamente pelo uso da Penicilina G Benzatina, o esquema terapêutico pode estar incorreto, justificando a persistência dos altos $\operatorname{casos}^{15,24,25,32}$. Dito isto, podemos estar diante de uma inadequação diagnóstica da assistência e, consequentemente, do registro das notificações, o que, por sua vez, pode justificar o elevado índice de dados ignorados nesta variável. Precisa-se, portanto, investir na qualificação dos profissionais para que a avaliação clínica e a assistência sejam mais assertivas e resolutivas.

O Ministério da Saúde preconiza a triagem sorológica para SF no PTG, TTG e durante a internação para o parto ou curetagem ${ }^{6,8,15,21}$. Desta forma, a SC nada mais é que um evento sentinela resultante de diversas oportunidades de prevenção desenvolvidas incorretamente, que se mantém com a persistência de diagnósticos tardios e tratamentos inadequados ${ }^{22,29}$.

Um estudo realizado nos anos de 2011 e 2014 na cidade do Rio de Janeiro registrou, dos 6.274 casos de SC mães com faixa etária entre 20 e 34 anos (62\%), ensino fundamental incompleto (31\%), realização de PN (73\%), diagnóstico materno de SF no momento do parto e curetagem (44\%) e durante o PN (43\%) e tratamento materno inadequado (52\%). O parceiro não foi tratado em $61 \%$ dos casos e grandes foram as taxas de itens ignorados30. Ainda que o Brasil possua cobertura PN superior a $95 \%$, a taxa de SC é um valioso indicador de assistência PN falha, desqualificada e desigual, pois conflita com os elevados registros de $\mathrm{SG}$ e $\mathrm{SC}^{22,25,27,32}$. Todavia, outros autores afirmam opiniões e resultados semelhantes com relação à alta porcentagem de PN realizados, tratamento inadequado, ignorado ou não realizado em gestantes e seus parceiros ${ }^{4,21,22,23,25,33}$. Portanto, espera-se que pesquisadores e gestores avaliem quais fatores estejam sendo incorretos ou inapropriados na cobertura PN ofertada para que novas estratégias sejam traçadas no intuito de complementar as deficiências e reduzir a incidência e prevalência da SF no estado, uma vez que seu diagnóstico e manejo clínico e terapêutico é simples e eficaz.

Importante ressaltar que, segundo Padovani, Oliveira e Pelloso (2018), as formas de se evitar e prevenir a SC vão além do tratamento medicamentoso apropriado para cada fase clínica da doença materna e seus contatos sexuais. Vários autores reafirmam que o objetivo só se pode ser alcançado com sucesso mediante triagem, busca ativa, monitoramento sorológico constante, diagnóstico precoce, vigilância epidemiológica, ações farmacológicas e laboratoriais e uma excelente atuação da equipe e dos prestadores de serviços em saúde $22,25,29,32,33$.

Uma pesquisa com dados globais da OMS registrou, no ano de 2008, 520 mil gestantes com SF materna, 215 mil natimortos e óbitos fetais, $90 \mathrm{mil}$ mortes neonatais, 150 mil crianças com SC e 21.500 mortes infantis ${ }^{34}$. Em todos os estudos observados os óbitos e abortos decorrentes da SC superaram e muito a meta nacional de 0,5 casos por mil NV estipulada para o ano de 2015 e até se mostraram superiores nos anos subsequentes ${ }^{4,11,21,22,24,25}$. Pelo que está registrado até agora, o coeficiente de mortalidade infantil por SC no Brasil foi de 6,1 e 8,2 óbitos por mil NV em 2016 e 2018, respectivamente ${ }^{4,22}$. Contudo, o estado do Rio de Janeiro, em 2018, apresentou taxa de 23,3, valor três vezes superior à média nacional ${ }^{4}$.

Estudo feito na capital carioca, entre 1999 e 2002, evidenciou redução da mortalidade perinatal por $\mathrm{SC}$, entretanto mortalidade específica estável ${ }^{30}$. Este estudo obteve dados que andam em consonância quase totalitária nas demais pesquisas levantadas, apesar de apresentar taxas de incidência e mortalidade por $\mathrm{SC}$ superiores na grande maioria observada ${ }^{21,22,24,27,30}$. Isto caracteriza um longo caminho a ser traçado e percorrido para que haja mudança do panorama em questão. Necessita-se cada vez mais do diagnóstico precoce e tratamento assertivo dos casos de forma que as complicações precoces e tardias como, por exemplo, abortos, natimortos, surdez neurológica, fronte olímpica e tíbia em lâmina de sabre sejam eliminadas ou minimizadas ${ }^{33}$.

As principais limitações deste estudo se relacionam, principalmente, à subnotificação e 
qualidade da informação, uma vez que se fez uso de dados secundários, ainda que sejam de fontes oficiais. Ademais, existe a possibilidade de erro, preenchimento incompleto e/ou incorreto das notificações, haja visto o elevado número de itens ignorados nas variáveis escolaridade, classificação clínica, raça e tratamento, o que pode subestimar os resultados.

\section{Considerações finais}

O estado do Rio de Janeiro se encontra em situação alarmante e inaceitável, pois os números de SA, SG e SC indicam falha e insuficiência significativa no controle da doença. É, portanto, de suma importância o conhecimento dos resultados apresentados para os municípios e estado elaborarem e intensificarem a aplicação de estratégias resolutivas à assistência materno-infantil no que tange a SF e suas repercussões. Reconhecer a realidade local da doença e combater as subnotificações estimulando o correto preenchimento das fichas de notificação também são ações positivas que devem ser constantemente cobradas e estimuladas, visando a fidedignidade das informações disponibilizadas pelos sistemas oficiais.

Fundamental também que recursos e estratégias sejam devidamente aplicadas no sentido de melhorar a qualidade dos pré-natais, investir em vigilância epidemiológica, capacitar os prestadores de serviço em saúde, ampliar o rastreamento e monitoramento dos prováveis casos, aumentar a adesão ao tratamento e manter o acesso e continuidade do atendimento de modo a colaborar para a redução das taxas de incidência e prevalência da doença. Desta forma, espera-se que a conformidade dos mecanismos contribua para o enfrentamento e controle deste agravo, minimizando consequentemente sua reemergência.

\section{Referências}

1. Andrade ALMB, Magalhães PVVS, Moraes MM, Tresoldi AT, Pereira RM. Diagnóstico tardio de sífilis congênita: uma realidade na atenção à saúde da mulher e da criança no Brasil. Rev. paul. pediatr. 2018;36(3):376381.

2. World Health Organization. Methods for surveillance and monitoring of congenital syphilis elimination within existing systems. Geneva: WHO; 2011

3. World Health Organization. Investment case for eliminating motherto-child transmission of syphilis: Promoting better maternal and child health and stronger health systems. Geneva: WHO; 2012.

4. Secretária de Vigilância em Saúde; Ministério da Saúde. Boletim Epidemiológico Sífilis 2019. Brasília, DF: O Ministério; 2019.

5. Tavares W, Marinho LAC. Rotinas de Diagnóstico e Tratamento das Doenças Infecciosas e Parasitárias. $4^{\mathrm{a}}$ Edição. São Paulo: Editora Atheneu; 2015 .

6. Ministério da Saúde. Manual técnico para diagnóstico da sífilis. Brasília, DF: O Ministério; 2016.
7. Ministério da Saúde; Secretaria de Vigilância em Saúde; Departamento de DST, Aids e Hepatites Virais. Sífilis: Estratégias para Diagnóstico no Brasil. Brasília, DF: O Ministério; 2010.

8. Ministério da Saúde; Secretaria de Vigilância em Saúde; Departamento de DST, Aids e Hepatites Virais. Protocolo Clínico e Diretrizes Terapêuticas para Atenção Integral às Pessoas com Infecções Sexualmente Transmissíveis. Brasília, DF: O Ministério; 2015.

9. Ministério da Saúde; Secretaria de Vigilância em Saúde; Programa Nacional de DST e Aids. Diretrizes para o Controle da Sífilis Congênita. Brasília, DF: O Ministério; 2005.

10. Secretaria de Estado de Saúde de São Paulo; Coordenadoria de Controle de Doença; Centro de Referência e Treinamento DST/Aids-SP, Programa Estadual DST/Aids-SP. Guia de bolso para o manejo da sífilis em gestantes e sífilis congênita. São Paulo, SP: Secretaria de Estado da Saúde; 2016.

11. Cavalcante PAM, Pereira RBL, Castro JGD. Sífilis gestacional e congênita em Palmas, Tocantins, 2007-2014. Epidemiol. Serv. Saúde. 2017;26(2):255-264.

12. Tiago ZS, Picoli RP, Graeff SV, Cunha RV, Arantes R. Subnotificação de sífilis em gestantes, congênita e adquirida entre povos indígenas em Mato Grosso do Sul, 2011-2014. Epidemiol. Serv. Saúde. 2017; 26(3): 503-512.

13. Barbosa KF, Batista AP, Nacife MBPSL, Vianna VN, Oliveira WW, Machado EL, et al. Fatores associados ao não uso de preservativo e prevalência de HIV, hepatites virais B e C e sífilis: estudo transversal em comunidades rurais de Ouro Preto, Minas Gerais, entre 2014 e 2016. Epidemiol. Serv. Saúde. 2019;28(2):e2018408.

14. Conceição HN, Câmara JT, Pereira BM. Análise epidemiológica e espacial dos casos de sífilis gestacional e congênita. Saúde debate. 2019;43(123):1145-1158.

15. Falavina LP, Lentsck MH, Mathais TAF. Tendência e distribuição espacial de doenças infecciosas em gestantes no estado do Paraná-Brasil. Rev. Latino-Am. Enfermagem. 2019;27:e3160

16. DATASUS. Informações de Saúde. Morbidade e informações epidemiológicas. Consulta online em agosto 2019. www.datasus.gov.br

17. Secretaria de Saúde do Estado do Rio de Janeiro. Atualização do Plano Diretor de Regionalização 2012/2013. Rio de Janeiro, RJ: Secretaria de Saúde do Estado do Rio de Janeiro; 2013.

18. Microsoft Corporation Microsoft Excel. Version 2016. Redmond: Washington, 2016.

19. Secretaria de Saúde do Estado do Rio de Janeiro [homepage na internet]. Assessoria de Regionalização: O Processo de Regionalização no Estado do Rio de Janeiro [acesso em 10 ago 2020]. Disponível em: https:// www.saude.rj.gov.br/

20. Instituto Brasileiro de Geografia e Estatística [homepage na internet]. Cidades e Estados [acesso em 10 ago 2020]. Disponível em: https://www. ibge.gov.br

21. Vescovi JS, Schuelter-Trevisol F. Aumento da incidência de sífilis congênita no estado de Santa Catarina no período de 2007 a 2017: análise da tendência temporal. Rev Paul Pediatr. 2020;38:e2018390.

22. Soares KKS, Prado TN, Zandonade E, Moreira-Silva SF, Miranda AE. Análise espacial da sífilis em gestantes e sífilis congênita no estado do Espírito Santo, 2011-2018. Epidemiol. Serv. Saúde. 2020;29(1):2018193.

23. Silva MJN, Barreto FR, Costa MCN, Carvalho MSI, Teixeira MG. Distribuição da sífilis congênita no estado do Tocantins, 2007-2015. Epidemiol. Serv. Saúde. 2020;29(2):e2018477.

24. Carvalho IS, Brito RS. Sífilis congênita no Rio Grande do Norte: estudo descritivo do período 2007-2010. Epidemiol. Serv. Saúde. 2014;23(2):287294.

25. Padovani C, Oliveira RR, Pelloso SM. Sífilis na gestação: associação das características maternas e perinatais em região do sul do Brasil. Rev. Latino-Am. Enfermagem. 2018;26:e3019.

26. Santos TD, Lock NC, Lnza SS, Santos AB, Beck ST, Anversa ETR et al. Perfil da sífilis gestacional e congênita no município de Santa Maria - 
RS: vivências multidisciplinares para troca de saberes. Saúde (Santa Maria). 2016;42(2):215-224.

27. Nascimento LFC. Sífilis congênita no Vale do Paraíba sob enfoque espacial. Rev Paul Pediatr. 2020;38:e2018395.

28. Arando M, Vall-Mayans M. The Epidemiological and Clinical Characteristics of the Epidemic of Syphilis in Barcelona. Actas DermoSifilográficas. 2019;110(10):841-849.

29. Bowen $\mathrm{V}, \mathrm{Su}$ J, Torrone E, Kidd S, Weinstock H. Increase in incidence of congenital syphilis - United States, 2012-2014. MMWR. 2015;64(44):1233-1260.

30. Reis GJ, Barcellos C, Pedroso MM, Xavier DR. Diferenciais intraurbanos da sífilis congênita: análise preditiva por bairros do Município do Rio de Janeiro, Brasil. Cad. Saúde Pública. 2018;34(9):e00105517.

31. Araujo LRL, Silva VCC, Gouveia Filho OS, Sousa MNA. Prevalência de sífilis gestacional e congênita no estado de Goiás, Brasil. C\&D-Revista Eletrônica da Fainor, Vitória da Conquista. 2016;(2):49-58.

32. Magalhães DMS, Kawaguchi IAL, Dias A, Calderon IMP. Sífilis materna e congênita: ainda um desafio. Cad. Saúde Pública. 2013;29(6):11091120.

33. Macêdo VC, Lira PIC, Frias PG, Romaguera LMD, Caires SFF, Ximenes RAA. Risk factors for syphilis in women: case-control study. Rev Saúde Pública. 2017;51:78.

34. Newman L, Kamb M, Hawkes S, Gomez G, Say L, Seuc A, et al. Global Estimates of Syphilis in Pregnancy and Associated Adverse Outcomes: Analysis of Multinational Antenatal Surveillance. PLOS Medicine. 2013;10(2):e1001396 